$\begin{array}{cl}\begin{array}{cl}\text { Revue } \\ \text { de /histoire }\end{array} & \text { Revue de l'histoire des religions } \\ \text { des religions } & \begin{array}{l}3 \mid 2006 \\ \text { Varia }\end{array}\end{array}$

\title{
Dionysos dans le Protreptique de Clément d'Alexandrie
}

Initiations dionysiaques et mystères chrétiens

Dionysus in Clement of Alexandria's Protrepticus

\section{Fabienne Jourdan}

\section{OpenEdition}

\section{Journals}

Édition électronique

URL : http://journals.openedition.org/rhr/5180

DOI : $10.4000 /$ rhr. 5180

ISSN : 2105-2573

\section{Éditeur}

Armand Colin

\section{Édition imprimée}

Date de publication : 1 septembre 2006

Pagination : 265-282

ISBN : 2200-92105-5

ISSN : 0035-1423

\section{Référence électronique}

Fabienne Jourdan, «Dionysos dans le Protreptique de Clément d'Alexandrie », Revue de l'histoire des religions [En ligne], 3 | 2006, mis en ligne le 25 janvier 2010, consulté le 20 avril 2019. URL : http:// journals.openedition.org/rhr/5180; DOI : 10.4000/rhr.5180 


\title{
Dionysos dans le Protreptique de Clément d'Alexandrie
}

\author{
Initiations dionysiaques \\ et mystères chrétiens
}

\begin{abstract}
Dans son Exhortation aux Grecs, Clément d'Alexandrie dénonce vigoureusement le caractère scandaleux des multiples figures de Dionysos. Il est néanmoins remarquable qu'il rattache cette divinité à tous les mystères païens dont Orphée serait l'initiateur. Le choix de concentrer presque exclusivement son attaque sur cette cible et ses avatars révèle sans doute son intention d'adapter la thématique dionysiaque aux mystères chrétiens. Mais Clément ose-t-il davantage? De même qu'il a converti Orphée dont la transfiguration scelle l'avènement du chantre christique, suggérerait-il implicitement un parallèle entre Dionysos et le Christ destiné à intégrer dans la sphère chrétienne un dieu fascinant mais insaisissable?
\end{abstract}

\section{Dionysus in Clement of Alexandria's Protrepticus}

In his Exhortation to the Greeks, Clement of Alexandria protests about the scandalous nature of Dionysus' numerous figures. Still it is noticeable that he links this divinity with all the pagan Mysteries which were said to be introduced by Orpheus himself. Clement chooses to focus his attack almost exclusively on this particular divine target and its avatars. Such a choice reveals that he is determined to adapt the dionysiac set of themes to the Christian Mysteries. However, does Clement go any further on that point? Just as he converted Orpheus and transfigured him, which was a way of sealing the advent of Christ conceived as a singer, does he not seem to imply a parallel between Dionysus and Christ in order to integrate such a fascinating but elusive god into the Christian sphere? 
Pour inviter les Grecs païens de sa cité à embrasser la religion chrétienne, Clément d'Alexandrie entreprend d'abord de les détourner de leurs propres traditions mythiques et cultuelles. Le Protreptique dénonce tous les aspects choquants qu'elles peuvent comporter aux yeux d'un homme cultivé de l'époque (II ${ }^{\mathrm{e}}-\mathrm{III}^{\mathrm{e}}$ ap. J.-C.). Bien que vraisemblablement composé à l'aide d'un manuel ${ }^{1}$, ce discours prétend « mettre à nu ${ }^{2}$ » les différents rites secrets afin d'en dévoiler l'inanité et l'immoralité. Dans la présentation qu'en donne l'apologiste ${ }^{3}$, ces derniers ont tous Orphée pour initiateur et se rattachent presque tous à la figure de Dionysos par le biais de ses multiples épiclèses. Pourquoi un tel choix ? Le dieu de l'ivresse et des processions phalliques constitue assurément une cible idéale pour qui veut condamner la luxure qui aurait caractérisé les initiations grecques. Il n'en demeure pas moins que Clément réadapte habilement tout le vocabulaire des mystères bacchiques dans son exhortation à se convertir au christianisme. Il adopte certes par là une rhétorique fidèle à la tradition littéraire inaugurée par Platon et reprise par le Juif Philon d'Alexandrie $^{4}$. Mais va-t-il jusqu'à faire de Dionysos une figure annonciatrice du Dieu unique, de la même façon qu'il a métamorphosé Orphée après l'avoir converti - en chantre préfigurant le Christ $^{5}$ ? Nombre

1. Voir Riedweg, 1987, p. 117.

2. En raison des contraintes de la publication, nous nous contentons ici de traductions et adoptons une translittération sans accent pour les mots indispensables.

§ II, 12, 1 : « Est-ce que je vous ferais la liste des mystères ? Je ne vais pas les parodier comme on dit que le fit Alcibiade, mais bien mettre à nu, laissant parler la vérité, la charlatanerie qui est cachée en eux ; oui, et ceux que vous appelez des dieux et auxquels s'attachent les initiations associées aux mystères, je vais les faire paraître eux-mêmes sur la scène des mœurs, devant les spectateurs de la vérité. » (Nous traduisons nous-même les textes de Clément d'Alexandrie cités.)

3. Ou l'auteur du manuel, bien qu'il soit possible de montrer combien Clément intervient lui-même dans cet exposé par les choix qu'il opère et sa manière de présenter chaque récit étiologique.

4. Voir Riedweg, 1987, p. 155sq.

5. J.-M. Roessli (2002) insiste sur l'opposition à l'œuvre chez Clément entre le pouvoir incantatoire d'Orphée et celui du Logos. Cette antithèse est présente, mais dépassée selon nous, grâce à la description du chant nouveau en termes empruntés à la légende du chant orphique et grâce à la citation du hieros logos attribué au chantre thrace à la fin du Protreptique. 
d'indices laissent penser que Clément a peut-être été tenté d'opérer semblable transfiguration. L'examen minutieux de la situation polémique dans laquelle s'inscrit son discours explique néanmoins l'impossibilité pour le chrétien de la réaliser.

\section{CONDAMNATION DES MULTIPLES FigURES DE DiONySOS}

Deux reproches sont adressés aux mystères païens : tous sont marqués du sceau de la luxure et ne sont que cérémonies funèbres. Chacun des récits relatifs à Dionysos dans le Protreptique donne prise à une telle accusation. Nous n'insisterons ici que sur son premier aspect. Aux yeux du polémiste chrétien qui se place dans la perspective biblique et refuse d'envisager la signification que peuvent avoir ces éléments dans la tradition religieuse païenne ${ }^{6}$, la prédominance du sexe dans les cultes et l'omniprésence du serpent incarnation du Mal qui induit à la luxure - sont hautement condamnables. Or, ce sont là les deux caractéristiques principales des mythes étiologiques attachés aux mystères ici considérés comme étant ceux de Dionysos. Passons-les rapidement en revue.

\section{Naissance de Dionysos}

La naissance du dieu constitue un premier récit donnant lieu à un rituel secret (§ II, 12, 3 ; § II, 16, 1). Zeus, identifié à Sabazios, l'engendre après un second viol et inceste, dans lequel il prend la forme d'un serpent. Les mystères rattachés à cet épisode font allusion à une union charnelle. Elle est mimée par le geste qui consiste à faire passer un serpent dans le giron de l'initié ${ }^{7}$.

6. Nous n'insisterons pas ici sur la signification du culte rendu au phallus et sur la symbolique du serpent dans la tradition religieuse grecque. Rappelons néanmoins que le premier y est signe de fécondité, et se voit en cela réellement digne de vénération. Le second est lié d'une part aux rites de fécondité en tant qu' animal considéré comme fécondant la Terre, d'autre part aux cultes funéraires en tant qu'incarnation de l'âme du mort ou protecteur des tombeaux (voir E. Küster, 1913, p. 68, 138, 149).

7. § II, 16, 1 : «Déméter enfante. Corè grandit. Et, de nouveau, celui qui l'avait engendrée, ce Zeus, s'unit à Phéréphatta, sa propre fille, après l'avoir fait avec sa mère Déo, oubliant la première souillure, lui, Zeus, père et corrupteur 


\section{Le sexe du défunt}

Après le récit de la mise à mort du jeune Dionysos, suit celui du meurtre du Corybante par ses deux frères. Le syncrétisme ici sans cesse à l'œuvre les assimile aux Cabires, et le sexe du défunt devenu objet de piété chez les Tyrrhéniens se révèle finalement être celui de Dionysos ${ }^{8}$. Dans le texte, Clément joue sur l'effet oratoire de la triple répétition du terme aidoia («parties honteuses ») pour stigmatiser un rituel qu'il juge licencieux.

\section{Iacchos}

Dionysos est peut-être aussi présent dans l'histoire de Déméter et Baubo à rattacher aux mystères d'Éleusis. Il apparaîtrait cette fois sous son épiclèse d' $\mathrm{d}^{\text {Iacchos }}{ }^{9}$ ». Clément explique que Baubo, face à la déesse qui, dans son chagrin, refuse de boire le cyceon, découvre ses parties honteuses ( $\S$ II, 20, 1-2). Démeter rit et accepte la coupe. Le terme « Iacchos », quant à lui, soit référerait à l'importun et trop jeune spectateur d'une scène impudique, soit serait une désignation du sexe auquel Baubo donne la forme d'un enfant dans la version du mythe transmise par Arnobe ${ }^{10}$. Clément

de la jeune fille ("corè"); et il s'unit à elle après être devenu un serpent, ce qui le dénonçait déjà. (2) En tous cas, dans les mystères de Sabazios, "le dieu qui passe par le giron" est une formule symbolique pour les initiés, et c'est un serpent que l'on tire dans le giron de ceux qui reçoivent l'initiation, geste qui dénonce l'intempérance de Zeus. (3) Phéréphatta enfante à son tour. Elle met au monde un fils qui a la forme d'un taureau. »

8. § II, 19, 4 : «Les deux fratricides, en effet, emportèrent la corbeille dans laquelle se trouvait le sexe de Dionysos pour l'amener en Tyrrhénie, colporteurs qu'ils étaient d'une glorieuse marchandise ! Ils restèrent là puisqu'ils étaient en exil, offrant aux Tyrrhéniens cette très vénérable leçon de piété : honorer comme objets divins une corbeille et des parties honteuses. C'est pour cette raison, et non sans vraisemblance, que certains veulent appeler Dionysos Attis parce qu'il s'est vu privé de ses parties honteuses. »

9. Voir aussi $§ 62,3$.

10. Les vers cités comportent en effet une difficulté examinée respectivement par Fr. Graf et M. Marcovich. Ils pourraient en effet être compris de deux manières. Soit ils signifient simplement que « l'enfant Iacchos était là » et indiquent la présence de Dionysos-Iacchos dans cette scène. Soit, selon la lecture d'Arnobe (Adv. nat. 5.26 : Sic effata simul vestem contraxit ab imo/ 
évoque encore un mythe et recourt à deux épiclèses destinées à confiner le dieu dans le domaine de la luxure.

\section{Une catabase de Dionysos}

Il assigne au récit de la descente de Dionysos aux Enfers la fonction d'expliquer les processions phalliques dans les cités (§ II, $342-5^{11}$ ). Prosymnos aurait indiqué au dieu le chemin de l'Hadès en échange de la promesse d'obtenir de sa part le plaisir charnel à son retour. Son «passeur» étant mort entre-temps,

obiecitque oculis formatas inguinibus res :/quas cava succutiens Baubo manu (nam puerilis/ollis vultus erat) plaudit, contrectat amice) présentée par F. Graf (1974, p. 196), ils suggéreraient que Baubo donne à son sexe la forme d'un « enfant » (pais) pour faire une plaisanterie. Dans son interprétation, Fr. Graf suit H. Diels («Arcana Cerealia», in Miscellanea di Archeologia, Storia, Filologia dedicata al Prof. A. Salinas, Palermo, 1907, p. 3) qui renvoyait à une glose d'Athénée (3, 98 D) selon laquelle iacchos pourrait signifier choiros (le « porcelet ») et rappelait que ce dernier terme, depuis les Acharniens d'Aristophane (v. 773, 781 etc.), a le sens obscène de « sexe féminin ». Mais après un nouvel examen du texte d'Athénée, M. Marcovich (1986, p. 294) soutient qu'en réalité jamais dans la littérature grecque iacchos n'a signifié choiros. Clément, en tout cas, ne semble pas percevoir ce sens. Certes, il ne mentionne pas non plus Dionysos dans sa paraphrase des vers. Mais comme il rattache d'une manière ou d'une autre tous les mystères à cette divinité, il est vraisemblable qu'il ait vu en «Iacchos » un nom propre. Le passage faisant du jeune dieu l'insolent témoin d'une scène impudique contribue à sa condamnation.

11. $\S \mathrm{II}, 34,2$ : «Eh bien voilà l'ignominie que répandent sur le monde entier les jeux publics et les phallus consacrés à Dionysos, un fléau qui infeste les mœurs. (3) Dionysos, en effet, désirait vivement descendre dans l'Hadès mais en ignorait le chemin. $<$ Un homme $>$, du nom de Prosymne, promit de le lui montrer, mais non sans réclamer un salaire. Or, ce salaire n'avait rien de convenable ; pour Dionysos cependant, il l'était. Il s'agissait de se procurer du plaisir charnel, tel était le salaire que Prosymne exigeait de Dionysos. Le dieu consentit à la demande. Il promit de le lui procurer s'il revenait de l'Hadès et confirma sa promesse par un serment. (4) Après avoir appris quel chemin prendre, il s'en alla. De retour, il ne retrouva plus Prosymne. En effet, il était mort. Pour s'acquitter consciencieusement de la promesse faite à son amant, Dionysos se hâta vers sa tombe plein d'un désir luxurieux. Après avoir coupé une branche de figuier qui se trouvait là, il confectionna une sorte de membre viril et s'assit sur la branche, accomplissant ainsi sa promesse à l'égard du mort. (5) Le mystère célébré en souvenir de cet épisode : des phallus consacrés à Dionysos que l'on dresse à travers les cités. » 
Dionysos s'acquitta néanmoins à l'aide d'une branche de figuier. La partialité du polémiste est remarquable. Il ne mentionne pas le but de cette descente aux Enfers et concentre uniquement son récit sur la prostitution à laquelle se livre le prétendu dieu. Jamais Clément ne cherche à comprendre le sens de ces anciens mythes qu'il déforme pour son propos ${ }^{12}$.

\section{Dionysos-Bassaros}

Dionysos apparaît encore dans le Protreptique sous le nom de « Bassaros » (§ II, 22, 4). Ses initiations sont alors caractérisées par la présence du serpent - instrument du Mal et de la luxure aux yeux du chrétien, et des grains de sel évocateurs du sperme. Cette épiclèse se rattache peut-être originellement à un substantif désignant le «renard », comme le propose Hésychius, et évoquerait la peau de cet animal portée par les Bacchantes. Il aurait évolué par la suite dans le sens licencieux qu'entend vraisemblablement Clément. Le lexique de Suidas donne en effet pornikos comme synonyme de bassarikos et définit la bassaris comme une hetaira, une pornè, c'est-à-dire « une courtisane, une prostituée ».

\section{Dionysos Choiropsale}

L'Alexandrin mentionne enfin Dionysos Choiropsale (§ II, 39, 4), " préposé aux parties féminines ${ }^{13}$ ». L'étymologie du nom suffirait à elle seule à condamner cette figure du dieu aux yeux d'un chrétien : choiros, le «porcelet », est une désignation du « sexe féminin »; le verbe psallein signifie quant à lui «tirer brin à brin, poil à poil». Un scholiaste aux Perses d'Eschyle expliquait qu'il était synonyme de tillein et donnait précisément cet exemple : «Dionysos Choiropsale, celui qui épile les parties des femmes ${ }^{14} »$.

12. Une interprétation possible serait que cette légende aurait été destinée à expliquer un rituel d'initiation des jeunes garçons, qui, après un acte d'autohumiliation, auraient atteint le statut d'adulte. Voir Casadio, 1992, p. 212.

13. § II, 39, 3: «Quant à Dionysos Choiropsale ("Qui épile le sexe des femmes") maintenant, le passerai-je sous silence ? Les Sicyoniens l'adorent après l'avoir préposé aux parties féminines, révérant dans l'instigateur de la démesure le gardien de l'ignominie. »

14. Schol. Aesch. Pers. 1062. 
La multiplicité des figures dionysiaques évoquées recouvre en réalité l'unité fondamentale de la condamnation. Sous toutes ses formes, le dieu, selon Clément, incite à la luxure. Il est l'allié du Mal. L'accusation est étayée par l'association définitive de ses initiations au personnage d'Ève. Trompée par le suppôt du Diable, elle est l'instigatrice de la chute du genre humain et devient à son tour, sous la plume de l'apologiste, une figure du serpent. Clément, en effet, n'hésite pas à transcrire le cri poussé par les Bacchantes, le fameux «Ehoé », en « Eva », et à expliquer que si ce nom reçoit l'esprit rude (Héva), il signifie « serpent femelle» (§ II, 12, $\left.2{ }^{15}\right)^{16}$. Mais pourquoi insiste-t-il à ce point sur la figure de Dionysos et de ses mystères dans sa dénonciation des insanités et immoralités du paganisme?

\section{Un CHOIX CENTRÉ SUR LA FIGURE DE DiONYSOS}

La tendance de Clément à ramener presque tous les rites initiatiques à la figure de Dionysos est frappante. Il donne même le terme de « souillure » (apo tou musous) qui réfère vraisemblablement à sa mise à mort comme étymologie du mot «mystère » ${ }^{17}$. Qu'il ait ou non emprunté sa présentation des cultes secrets païens à un manuel, ce choix polémique lui appartient. La diversité des figures du Dieu recouvre une seconde unité après celle de la condamnation : celle de la vision du chrétien sur les mystères païens. Pourquoi ce choix

15. § II, 12, 2 : «C'est Dionysos devenu fou que célèbrent les Bacchants dans leurs rites, mangeant de la chair crue dans leur délire sacré. Couronnés de serpents, ils partagent rituellement la chair des victimes, en poussant un grand cri : "Eua" - cette Eva responsable de la faute qui lui est associée. Le signe des rites bacchiques est un serpent qui intervient dans l'initiation. De fait, maintenant, d'après la prononciation rigoureuse des Hébreux, le nom d'Héva reçoit l'esprit rude et signifie "serpent femelle". »

16. Wolfram Kinzig me fait remarquer que cette interprétation est encore défendue de nos jours par les exégètes de l'Ancien Testament (voir Claus Westermann, Genesis, commentaire à Gen. 3, 20).

17. § II, 13, 1 : «Il me semble qu'il faut aussi donner l'étymologie des mots "orgies" et "mystères". Le premier vient de la colère (orgè) que Déo ressentit contre Zeus, le second de la souillure (musos) qui eut lieu autour de Dionysos. » La souillure renvoie sûrement au meurtre du dieu par les Titans, mais pourrait aussi correspondre à l'inceste à l'origine de sa naissance. 
de Dionysos? Une saisie des traits communs à tous ces rituels aurait pu suffire à les dénigrer. Une réponse peut être cherchée dans plusieurs directions.

\section{Un choix historique?}

Il est peu vraisemblable que Clément s'en prenne réellement à une réalité cultuelle de l'époque ou du lieu. L'influence des rituels centrés sur les figures d'Isis et d'Osiris, identifiés à Déméter et Dionysos par un Plutarque aurait pu fournir une explication - mais l'apologiste n'y fait qu'à peine allusion ${ }^{18}$. Sa matière est toute littéraire et se veut exhaustive. Elle ne semble en rien relever d'une connaissance concrète des phénomènes décrits. L'Alexandrin est sans doute beaucoup plus imprégné de représentations théâtrales des Bacchantes.

\section{Un choix rhétorique}

Ce choix correspond avant tout à la rhétorique propre au Protreptique. Ce discours, avons-nous dit, tend à élever les païens à la religion du Dieu unique. Pour séduire ses destinataires, il utilise un matériau qui leur est familier, mais qu'il a d'abord dégagé de sa gangue païenne en en condamnant les traits licencieux. Les paragraphes X, 119, 1-120, 1 montrent l'aboutissement de cette rhétorique d'exhortation ${ }^{19}$. Elle opère la conversion chrétienne des orgies

18. $\S 48$.

19. Retenons seulement un passage caractéristique de ce travail de refonte, le paragraphe XII, 119, 1 : «Voici la montagne aimée de Dieu. Elle n'est pas le siège de tragédies contrairement au Cithéron, mais elle est consacrée aux drames de la vérité, montagne où règne la sobriété, ombrage des forêts sacrées. Les Bacchanales n'y sont pas célébrées par les "sœurs de Sémélé la foudroyée", par les Ménades initiées au partage impur des chairs, mais par les filles de Dieu, les belles agnelles dont les oracles révèlent les vénérables orgies en l'honneur du Logos et qui se rassemblent en un chœur plein de sagesse. (2) Le chœur, ce sont les justes. Le chant, un hymne au roi de toutes choses. Les jeunes filles font vibrer la lyre, des anges célèbrent la louange, des prophètes parlent, la musique commence à retentir, ils courent en un thiase, ils se hâtent, les appelés, dans leur désir de recevoir le Père. » 
bacchiques. Clément réutilise ici le motif littéraire des mystères devenu classique depuis Platon et entré dans le domaine judéochrétien avec Philon ${ }^{20}$. Mais il est remarquable que ce ne sont pas seulement les mystères d'Éleusis permettant de développer la métaphore de la lumière et de la vision divine qu'il réintègre à sa sphère de croyance. Clément fait aussi entrer dans le chœur chrétien les obscures orgies de Dionysos. Il est évident que ce dieu l'intéresse tout particulièrement. Il faut noter que cette utilisation du vocabulaire des initiations dionysiaques transforme complètement la visée des mystères païens, leur conférant une dimension spirituelle qui leur faisait défaut et leur prêtant une perspective de changement radical dans l'attitude religieuse qu'ils n'avaient pas. Comme l'a montré W. Burkert ${ }^{21}$, l'appropriation chrétienne du lexique des mystères antiques est responsable des clichés encore véhiculés de nos jours à leur sujet.

\section{Un choix théologique et littéraire}

Dionysos surtout est le fils de Zeus et dans une perspective chrétienne comme celle de Clément, il aurait pu être rapproché du Christ, Dieu, Fils de Dieu. La similitude était à ce point perceptible que Justin était allé jusqu'à prétendre que les démons avaient inventé des fils à Zeus et leur avaient attribué des actions semblables à celles du Christ afin de reprocher au christianisme de n'être qu'une pâle imitation de la religion païenne. Le cas de Dionysos lui semblait le plus évident : ne disait-on pas de l'inventeur de la vigne qu'il était lui aussi monté au ciel après avoir été mis en pièces ${ }^{22}$ ?

20. Riedweg, 1987.

21. Burkert, 2003, p. 5.

22. Justin, Première Apologie, $§ 54,2$ : «Ceux-ci, en effet, sachant par les prophètes que le Christ devait venir et que les impies seraient punis par le feu, entreprirent de faire attribuer à Zeus un grand nombre de fils, car ils comptaient bien pouvoir obtenir ainsi que les hommes considèrent tout ce qui concerne le Christ comme une invention mensongère et du même acabit que les fables racontées par les poètes. [...] (5) Ainsi le prophète Moïse [...] avait fait la prophétie suivante : "Il ne manquera pas de prince de la descendance de Juda, ni de chef de sa race, jusqu'à ce que vienne celui à qui il est réservé ; il sera dans l'attente des nations, il attachera son poulain à la vigne et lavera sa 
C'est tout d'abord le Dionysos d'Euripide bien connu de Clément qui aurait pu retenir son attention s'il avait souhaité établir un tel parallèle. Dans les Bacchantes, Dionysos est présenté comme le dieu nouveau. Il est l'étranger qui entre dans la cité de Thèbes, comme Jésus de Nazareth à Jérusalem. Il est Fils de Zeus et d'une mortelle, Sémélée 23 , dont les sœurs n'ont pas voulu croire que l'enfant était réellement rejeton du dieu ${ }^{24}$. La naissance de Jésus n'est pas sans rappeler celle-ci. Pour venir révéler sa divinité aux hommes ${ }^{25}$, Dionysos prend forme humaine ${ }^{26}$. Mieux, dans les Bacchantes, sa personne se dédouble. Il est à la fois le dieu et son jeune prêtre. Porteur d'une loi nouvelle, il tend à la répandre ${ }^{27}$. Mais il fait nécessairement l'objet de l'incrédulité et des brimades. Penthée le condamne au châtiment en des termes auxquels peuvent faire penser

robe dans le sang de la grappe." (6) Comme ils avaient eu connaissance de ces paroles prophétiques, les démons racontèrent que Dionysos était né fils de Zeus et ils transmirent qu'il aurait découvert la vigne ; ils marquèrent la place du vin dans ses mystères et enseignèrent qu'il était monté au ciel après avoir été mis en pièce. » (Traduction de Charles Munier, Saint Justin. Apologie pour les chrétiens. Edition et traduction (Paradosis 39), Fribourg, 1995, à l'exception de la dernière phrase. Ch. Munier adopte en effet la leçon onon (« âne ») proposée par F. Sylburg (Heidelberg, 1593) à laquelle nous préférons la leçon oinon («vin») du cod. Parisinus gr. 450, datant de 1364.)

23. Bacchantes, v. 1-3: «Me voici, fils de Zeus, sur la terre thébaine, Dionysos, celui que jadis enfanta la fille de Kadmos, Sémélé, par le feu de la foudre accouchée » (traduction de H. Grégoire et J. Meunier, Paris, Les Belles Lettres, 1998).

24. Bacchantes, v. 26-27 : «Car les sœurs de ma mère, qui, plus que personne, auraient dû m'épargner cette insulte, ont affirmé que moi, Dionysos, je n'étais pas le fils de Zeus » (traduction de H. Grégoire et J. Meunier, Paris, Les Belles Lettres, 1998).

25. Bacchantes, v. $41:$ :En me manifestant aux hommes comme le Dieu qu'elle enfanta pour Zeus » (traduction de H. Grégoire et J. Meunier, Paris, Les Belles Lettres, 1998).

26. Bacchantes, v. 4-5: «J'ai pris la forme humaine pour venir aux sources de Dirkè aux eaux de l'Isménos » (traduction de H. Grégoire et J. Meunier, Paris, Les Belles Lettres, 1998).

27. Bacchantes, v. 447-49: «Mais je saurai lui [à Penthée] prouver et prouver au peuple entier de Thèbes que je suis né Dieu. Puis, ayant tout réglé à mon gré dans ces lieux, je porterai mes pas dans une autre contrée ; et partout en chemin je me ferai connaître » (traduction de H. Grégoire et J. Meunier, Paris, Les Belles Lettres, 1998). 
les scènes précédant la mise en croix, lorsqu'il lui dit par exemple : « tu seras châtié de tes méchants sophismes » (v. 488). On trouve de même dans les Actes des Apôtres une expression qui semble tirée de cet épisode des Bacchantes ${ }^{28}$. La catabase de Dionysos évoquée par Clément pourrait en outre, toutes proportions gardées, être rapprochée de celle d'Orphée à la quête d'Eurydice. Si elle n'était pas relatée de manière aussi burlesque, elle aurait autorisé un nouveau parallèle entre ce dieu, son poète et le Christ lui aussi descendu dans le royaume des morts.

Avec la figure du Dionysos des Bacchantes, Clément disposait déjà d'une préfiguration possible du Christ à présenter aux païens comme il l'avait fait dans le cas d'Orphée. Sous la plume de l'apologiste, en effet, ce "charlatan» était devenu une figure annonciatrice du chantre christique élevant la louange à Dieu. Le Dionysos des mystères - dieu de la fertilité, dieu salvateur et dieu chtonien - aurait également pu l'intéresser à titre théologique.

\section{Un choix théologique et cultuel?}

Dionysos à lui seul aurait été susceptible de préfigurer nombre des attributs qui reviennent en définitive au Dieu de la Bible, et ce, mieux que Zeus par exemple à une époque où les initiations aux mystères semblent avoir acquis plus d'importance que les cultes rendus aux Olympiens. Clément aurait pu réutiliser les motifs qui caractérisent cette divinité païenne. Jamais pourtant il ne décrit les pouvoirs que lui reconnaissaient les anciens Grecs, contrairement à ce qu'il fait pour Orphée explicitement intégré au chœur judéochrétien par le biais de son prétendu hieros logos élevé à la louange du Dieu de l'Ancien Testament ${ }^{29}$. Il ne s'agit pas de forcer le silence de l'apologiste, mais simplement de noter que dans les premiers siècles de notre ère, certains auteurs chrétiens, frappés par la similitude entre leur culte et les mystères, ont dénoncé ces derniers comme étant des contrefaçons diaboliques de la vraie

28. Actes des Apôtres, 26, 14 : " Il est dur pour toi de regimber contre l'aiguillon », cf. Bacchantes v. 795.

29. $\$ 74$. 
religion ${ }^{30}$. Même si les rites secrets des Grecs ne sont pas à rapprocher en eux-mêmes des pratiques du christianisme, ce dernier a parfois adopté leur imagerie, suivant en cela une tradition inaugurée par Platon. Clément s'adonne à la même adaptation. Mais Dionysos, le dieu libérateur, pouvait-il devenir chez lui une figure annonciatrice du Dieu unique ? Se trouvant au centre de mystères aisément transposables à la perspective chrétienne, cette divinité païenne aurait peut-être été plus à même que Zeus par exemple de remplir un tel rôle ${ }^{31}$.

Dans son Protreptique, Clément d'Alexandrie s'approprie totalement la thématique et le vocabulaire propres aux initiations dionysiaques. Mais franchit-il le pas qui consisterait à intégrer Dionysos lui-même à la sphère chrétienne, sinon le Dionysos d'Euripide, le Dionysos « orphique » auquel faisait allusion la citation de Justin?

\section{CléMENT INTÈGRE-T-IL LA FIGURE DU DIONYSOS « ORPHIQUE » AUX MYSTÈRES CHRÉTIENS ?}

Dionysos dans le chœur chrétien?

Pour Clément, la tentation a sans doute été grande d'intégrer Dionysos au chœur chrétien. Il est aisé de le comprendre si l'on remarque qu'il choisit de concentrer son attention sur une certaine figure du dieu, celui dont le «prêtre » serait Orphée ; celui qui, encore enfant, fut mis à mort par les Titans.

\section{Dionysos et Orphée}

La figure de Dionysos intéresse sans doute l'Alexandrin parce que le dieu païen est à la fois conducteur de rituels et fils de Zeus. Justin cependant nommait d'autres fils de Zeus qui pouvaient être

30. Voir n. 21 et Burkert, 2003, p. 5 et n. 11, p. 116.

31. Aristobule remanie précisément les premiers vers des Phénomènes d'Aratos en remplaçant le nom de Zeus par celui de « Dieu ». Voir Eusèbe de Césarée, Préparation Evangélique, XIII, 12, 6-7. 
rapprochés abusivement du Christ comme Persée par exemple, lui aussi né d'une vierge ${ }^{32}$. De plus, comme l'explique W. K. C. Guthrie, les points communs couramment relevés entre Dionysos et le Christ - filiation divine, souffrance, mort et résurrection - étaient caractéristiques du paganisme finissant. "Ils étaient vrais aussi d'Osiris et d'Adonis ou d'autres dieux tout aussi connus dans le monde gréco-romain que le Dionysos orphique, sinon davantage ${ }^{33}$. » Pourtant, selon nous, ce qui intéresse plus particulièrement Clément dans le Dionysos «orphique», c'est précisément son lien à Orphée. Le couple Dionysos-Orphée confère une unité à la pensée de l'apologiste. Dans le Protreptique, il s'est emparé du fondateur d'une religion nouvelle. Pour le convertir, il lui a fait chanter le hieros logos à la louange du Dieu unique. Il l'a présenté par ailleurs comme le hiérophante de toutes les initiations, et par conséquent aussi des orgies bacchiques. En unissant ces deux traits, il a peutêtre été tenté de donner au dieu de ce poète préfigurant le chantre christique le rôle de Dieu précisément. L'a-t-il fait ?

\section{La mort de Dionysos}

Son récit de la mise à mort de Dionysos devrait nous renseigner (§ II, 17, 2-II, 18, 134).

32. I, Apol., § 54 .

33. "They are commonplaces of the gods of the decline of paganism. They are true of Osiris and Adonis and many other god who was at least as well known in the Graeco-Roman world as the Orphic Dionysos, if not better», 1952, p. 267.

34. § II, 17, 2: «Quant aux mystères de Dionysos, ils sont absolument inhumains. Alors qu'il était encore enfant, les Courètes dansaient autour de lui, en armes, quand les Titans s'approchèrent sous le couvert d'une ruse. Après l'avoir trompé avec des jouets d'enfant, ces Titans précisément le mirent en pièces, bien qu'il fût encore tout petit, comme le dit le poète de l'initiation, le Thrace Orphée: "Un cône, un rhombe, des poupées articulées/De belles pommes d'or venues du jardin des Hespérides à la voix claire." (§ II, 18, 1) Et il n'est pas vain de vous présenter les vains symboles de cette initiation afin de la condamner : un osselet, une balle, une toupie, des pommes, un rhombe, un miroir, un flocon de laine. Cela étant, Athéna s'empara du cœur de Dionysos et en prit le nom de Pallas parce que le cœur palpitait encore. Quant aux Titans, qui étaient allés jusqu'à mettre en pièces Dionysos, ils placèrent un chaudron 
L'insistance sur la souffrance injuste d'un dieu encore enfant par le biais des expressions : «étant enfant », « avec des jouets d'enfant » et «alors qu'il s'agissait encore d'un tout petit enfant» (§ II, 17, 2), pouvait suggérer une comparaison avec le supplice infligé à celui qui est aussi représenté comme l'« enfant Jésus ». La notion de pathos, définie comme "souffrance », permettait de mieux encore tisser le lien. Si, pour un Grec païen, le terme faisait référence à l' " aventure » - ou, en l'occurrence, à la « mésaventure »- subie par Dionysos, pour un chrétien, il renvoyait à une passion, et en particulier à la Passion du Christ $^{35}$. Les deux figures divines auraient pu être ainsi aisément rapprochées par l'apologiste grâce à un pathos intolérable dans les deux cas, même s'il ne réfère pas à la même expérience selon que l'on se place dans une sphère religieuse ou l'autre.

Pourtant, dans ce même récit, même si tout est préparé pour un repas, Dionysos n'est pas mangé. Zeus intervient avant pour sauver son fils et permettre ainsi à son corps d'être enterré. Si Clément avait souhaité établir un parallèle entre le Dieu mangé et le Christ dont le corps est mangé par les fidèles à chaque Eucharistie, il aurait peut-être laissé les Titans le dévorer, à condition bien entendu que cet épisode du mythe fût connu de lui ${ }^{36}$. Or, jamais il ne tisse de luimême le rapprochement attendu.

sur un trépied, y jetèrent ses membres et commencèrent par les faire bouillir. Ils le "transpercèrent" ensuite avec de petites broches et le "tinrent au-dessus d'Héphaistos". (2) Mais, peu après, Zeus apparut. (S'il s'agissait d'un dieu, je suppose qu'il prit sans doute sa part au fumet des chairs rôties dont vos dieux avouent "recevoir le présent".) De son foudre il fit outrage aux Titans puis confia à son fils Apollon le soin d'ensevelir les membres de Dionysos. Celuici, qui n'avait garde de désobéir à Zeus, emporta le cadavre déchiré sur le Parnasse pour l'y enterrer. »

35. On ne peut s'empêcher ici de penser au centon d'Euripide, précisément intitulé Christus patiens attribué faussement à Grégoire de Nazianze et généralement tenu pour une œuvre byzantine médiévale. Il met en scène la passion et la mort du Christ dans une pièce de 2602 vers iambiques dont la moitié est empruntée au dramaturge grec.

36. Nous pouvons douter que Clément ait connu la version du mythe où Dionysos est dévoré. Rappelons par exemple qu'Athénagore qui veut dénoncer le cannibalisme des païens pour leur renvoyer les accusations qu'ils adressent aux chrétiens ne mentionne pas cet épisode alors qu'il cite nombre de passage, appartenant à une version de la théogonie orphique. Il est possible qu'il l'ignore tout simplement - si tant est que l'on puisse parler ici de cannibalisme - et que ce soit également le cas de Clément. Sur ce sujet, voir Jourdan, 2005. 
L'appropriation totale de la figure de Dionysos et de ses mystères aurait été tout aussi essentielle à l'apologiste chrétien que celle d'Orphée pour élever la parfaite louange au «chant nouveau». Mais bien qu'il ait sans doute été tenté de la réaliser, Clément d'Alexandrie ne propose pas de voir en Dionysos une préfiguration du Dieu chrétien. Un examen minutieux de la situation polémique dans laquelle s'inscrit son discours fournit l'explication de cette impossibilité. Les païens cultivés en effet établissaient d'eux-mêmes ce parallèle, et ce parfois pour dénoncer la vanité de la figure christique.

\section{Impossibilité d'intégrer la figure de Dionysos}

Dionysos et le Dieu chrétien chez Plutarque (et Valère Maxime ?)

Dans ses Questions de table ${ }^{37}$, Plutarque opère un rapprochement entre Sabazios et le Dieu juif par le biais, entre autres, de la proximité sonore qui existe entre les noms «Sabbah», «Sabbaoh» et «Sabazios ». Or, chez lui, Sabazios est un avatar de Dionysos $(671 \mathrm{~F})^{38}$. Le texte de Plutarque se ferait ainsi l'écho d'une confusion opérée à son époque par les païens. Elle serait peut-être également apparue chez Valère Maxime (I, 3,3) qui aurait expliqué que les Romains, en 139 ap. J.-C., auraient chassé les Juifs pour avoir introduit le culte de Sabazios à Rome. Nous resterons néanmoins prudente avant d'évoquer cet éventuel témoignage de Valère Maxime puisque E. N. Lane a montré que ce texte était vraisemblablement le fruit d'une reconstruction fautive à partir de plusieurs manuscrits ${ }^{39}$. À supposer que

37. IV, 6.

38. L'Athénien qui interrompt Symmache dans le dialogue souligne en effet que beaucoup appellent les Bacchants des «Sabi » (671 F). Il établit ensuite de nombreux parallèles destinés à expliquer comment le dieu national de Thèbes peut être enrôlé dans les mystères hébreux $(671 \mathrm{C})$. La fête des Tabernacles est ainsi comparée aux Dionysies (671 C). Les caractéristiques du Sabbah seraient les processions où l'on porte le thyrse et où le prêtre est vêtu en Bacchant (671 E, 672 A), l'utilisation de trompettes semblables à celles des fêtes dionysiaques à Argos (671 E), les quantités de vin absorbées (672 A). Il s'agirait d'une cérémonie nocturne à rapprocher des nuktelia bacchiques (672 B).

39. E. N. Lane, 1979. 
Clément ait eu écho d'une telle confusion entre son Dieu, Dionysos et/ou Sabazios, il aurait sans doute souhaité la récuser.

\section{Dionysos et le Christ chez Celse}

Mais il est encore plus intéressant de noter que le rapprochement entre Dionysos et le Christ a été opéré par un ennemi des chrétiens en vue de dénoncer la vaine prétention de Jésus au rang de Dieu. D'après Origène, dans son Discours vrai, Celse ${ }^{40}$ compare à la Passion la scène des Bacchantes où le prêtre de Dionysos, qui est Dionysos lui-même déguisé, est maltraité par Penthée. Le polémiste païen rappelle alors en citant un vers d'Euripide que, contrairement au Christ, le jeune homme avait la certitude de pouvoir être délivré par son dieu quand il le désirerait ${ }^{41}$. Il ajoute que Pilate ne fut pas puni contrairement à Penthée ${ }^{42}$ : les dieux traditionnels se vengent de leurs blasphémateurs ${ }^{43}$. Cela suffit à prouver selon Celse que le Christ n'est pas le fils d'un dieu. Sans compter par ailleurs que la souffrance est perçue par les philosophes païens comme indigne d'un dieu. Si elle suffit à faire déchoir Dionysos au rang de démon chez le médio-platonicien Plutarque ${ }^{44}$, elle contribue vraisemblablement pour Celse à dénier le rang divin au Christ.

Nous ne pouvons savoir si Clément connaissait les textes auxquels nous venons de nous référer. Mais il est probable qu'il ait eu des échos de telles critiques. Justin avait du moins déjà fait

40. Je dois à une remarque de Christoph Riedweg d'avoir pensé à Celse. Que lui soit exprimée ici toute ma reconnaissance.

41. Origène, Contre Celse, II, 34 : « le Juif de Celse qui croit railler Jésus est présenté comme s'il connaissait le mot de Bacchus chez Euripide (v. 498) : "Le dieu lui-même me délivrera quand je voudrai." » (traduction de Marcel Borret, in SC n 132, Paris, Cerf, 1967, p. 367).

42. Ibid. : " mais celui qui le condamna, dit-il, n'a rien souffert du sort de Penthée, pris de transport furieux et mis en pièces » (p. 368).

43. Ibid., VIII, 41 : « mais si tu avais insulté Dionysos lui-même ou Héraclès en personne, tu ne t'en serais peut-être pas tiré à si bon compte. Ton Dieu, on l'a torturé et crucifié en personne, et les auteurs du forfait n'ont rien eu à souffrir, pas même dans la suite de leur vie. [...] Nos dieux, du moins, se vengent sévèrement du blasphémateur, réduit pour cela à fuir et se cacher ou à être pris et mis à mort » $\left(\mathrm{SC} \mathrm{n}^{\circ} 150,1969\right.$, p. 263).

44. Isis et Osiris, 25, $360 \mathrm{E}$. 
remarquer que la similarité perçue à son époque entre le mythe de Dionysos et l'histoire du Christ était suffisamment forte pour constituer un danger. Il lui semblait nécessaire de mettre en garde contre une éventuelle confusion entre ces deux représentations du fils souffrant de Dieu ${ }^{45}$. Clément ne pouvait donc pas raisonnablement utiliser de son côté une comparaison dont ses adversaires se servaient pour condamner le Christ. Le seul rapport entre Dionysos et la Bible demeure chez lui confiné au domaine du mal. L’Evoé résonne définitivement comme le nom Eva.

Tout au long du Protreptique, Clément d'Alexandrie a travaillé à la conversion d'Orphée. Ainsi transfiguré, le chantre mythique est devenu chez lui une préfiguration du Christ, chanteur de la louange éternelle à Dieu. Mais bien qu'il ait sans doute été tenté d'opérer la même métamorphose sur le dieu d'Orphée, Dionysos, la situation polémique dans laquelle s'inscrivait son discours a vraisemblablement empêché l'apologiste de franchir ce pas. Il ne pouvait user d'une comparaison dont se servaient Celse et les ennemis du christianisme dans le but de nier la divinité de Jésus, Dieu, fils de Dieu.

Il n'en demeure pas moins que nous devons à Clément, qui est souvent notre principale source d'information concernant certains éléments rituels, une vision devenue traditionnelle des mystères païens. Sa description des cérémonies chrétiennes par le biais du vocabulaire des initiations dionysiaques a investi pour longtemps ces dernières d'une dimension spirituelle et d'une perspective de conversion religieuse qu'elles n'avaient pas en elles-mêmes ${ }^{46}$. Seule une analyse rigoureuse des autres sources peut corriger cette conception erronée du phénomène antique dont Clément est en grande partie responsable ${ }^{47}$.

\section{7 rue du 14 juillet \\ F - 28000 Chartres}

45. Guthrie, 1952, p. 267.

46. W. Burkert, 2003, p. 5.

47. Cette étude a d'abord fait l'objet d'une communication lors du colloque Orfeo y el orfismo. Nuevas pespectiva/Orpheus and Orphism: New approaches, organisé par Alberto Bernabé à Palma de Majorque du 3 au 5 février 2005. 


\section{BIBLIOGRAPHIE}

BURKERT Walter, Les cultes à Mystères dans l'Antiquité, (Ancient Mystery Cults, 1987) nouvelle traduction de l'anglais par Alain-Philippe Segonds, Paris, Les Belles Lettres, 2003.

CAsadio Giovanni, « Préhistoire de l'initiation dionysiaque », in L'Initiation, Actes du colloque international de Montpellier 11-14 avril 1991, T. I : Les rites de l'adolescence et les mystères, éd. par A. Moreau, Publications de 1'Université Paul Valéry, Montpellier, 1992, p. 209-213.

GRAF Fritz, «Baubo und ihr Anasyrma », Eleusis und die orphische Dichtung Athens in vorhellenistischer Zeit, Berlin, New-York, Walter de Gruyter, 1974, p. 194-199.

GutHRIE William Keith Chambers, Orpheus and Greek religion, Methuen \& Co., Londres, [1935] 1952², p. 264-271 (voir aussi la traduction française publiée chez Payot, Lausanne, 1956 et la réimpression publiée par Princeton University Press, Princeton, 1993).

JouRdan Fabienne, «Manger Dionysos. L'interprétation du mythe du démembrement par Plutarque a-t-elle été lue par les néo-Platoniciens ? », Pallas, n 67,2005 , p. 153-174.

KÜSTER Erich, Die Schlange in der griechischen Kunst und Religion, Giessen, Alfred Töpelmann, 1913.

LANE Eugène N., «Sabazius and the Jews in Valerius Maximus: A Reexamination », in The Journal of Roman Studies, LXIX, Londres, 1979, p. 35-38.

Marcovich Miroslav, «Demeter, Baubo, Iacchus, and a redactor », in Vigiliae Christianae, A Review of Early Christian Life and Language, Leiden, E. J. Brill, 1986, p. 295-299.

RIEDWEG Christoph, Mysterienterminologie bei Platon, Philon und Klemens von Alexandrien, Berlin, New-York, Walter de Gruyter (Untersuchung zur antiken Literatur und Geschichte, Band 26), 1987, p. 116-159.

RoEssLi Jean-Michel, «Convergence et divergence dans l'interprétation du mythe d'Orphée, de Clément d'Alexandrie à Eusèbe de Césarée », in Revue de l'histoire des religions, 219, 2002, p. 503-513. 